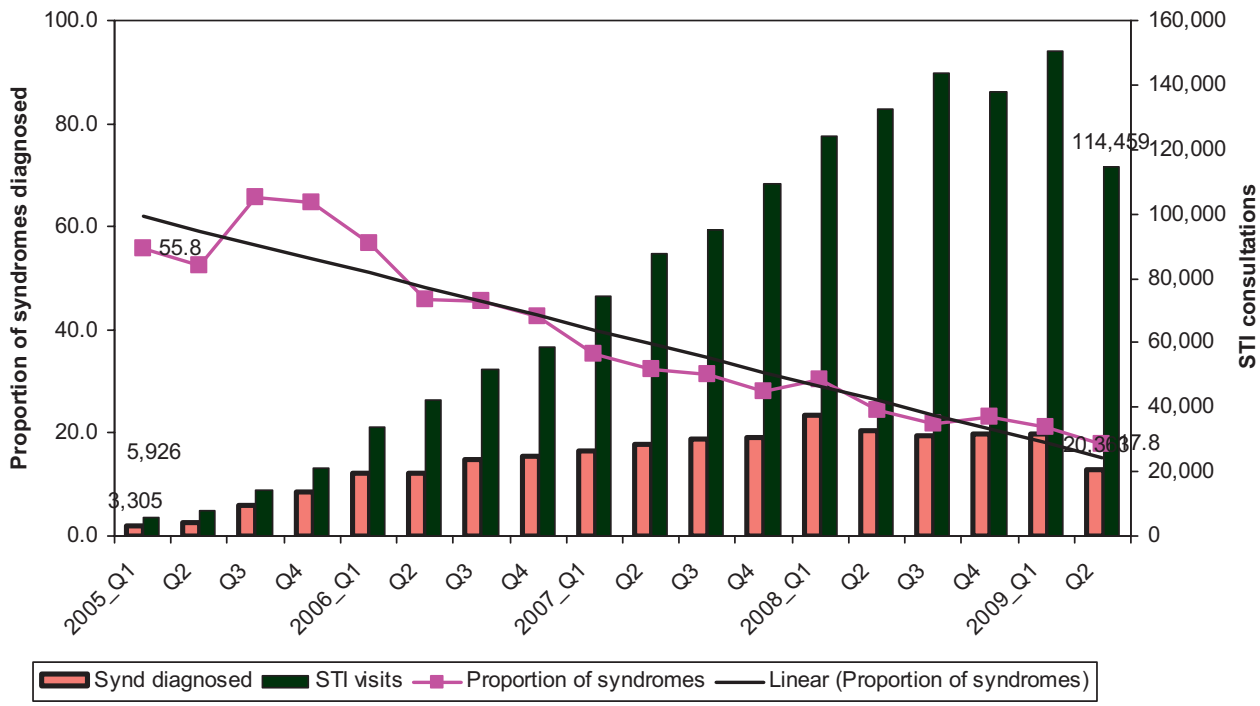

Abstract P5-S3.03 Figure 1 Decreasing trends of STI syndromes in the Avahan clinics Jan 2005 to June 2009.

clinics within 2 days of the onset of STI-related symptoms and acceptability of speculum and proctoscope examination increased significantly $(\mathrm{p}<0.001)$ see Abstract P5-S3.03 figure 1 .

Conclusions The project demonstrated that acceptable and accessible services with marginalised and often difficult-to-reach populations can be brought to a very large scale using standardised approaches. The paper presents the utilisation of these services, improved health seeking behaviour and reduced STI syndromes among the HRG.

\section{P5-S3.04 CONTINUED DECLINE IN GENITAL WARTS 3 YEARS AFTER INTRODUCTION OF OUADRIVALENT HUMAN PAPILLOMAVIRUS (HPV) VACCINATION PROGRAM}

doi:10.1136/sextrans-2011-050108.540

${ }^{1} \mathrm{~T}$ Read, ${ }^{1} \mathrm{~J}$ Hocking, ${ }^{1} \mathrm{~L}$ Gurrin, ${ }^{2} \mathrm{M}$ Chen, ${ }^{3} \mathrm{~B}$ Donovan, ${ }^{2} \mathrm{C}$ Bradshaw, ${ }^{1} \mathrm{C}$ Fairley. ${ }^{1}$ University of Melbourne, Carlton, Australia; ${ }^{2}$ Alfred Hospital, Australia; ${ }^{3}$ University of New South Wales, Australia

Background Vaccination of girls and young women aged 12-26, for HPV genotypes 6, 11, 16 and 18, commenced in Australia in 2007. Vaccine uptake is estimated to be $70 \%$.

\section{Presentations with Warts Quarters 2004 to end 2010}

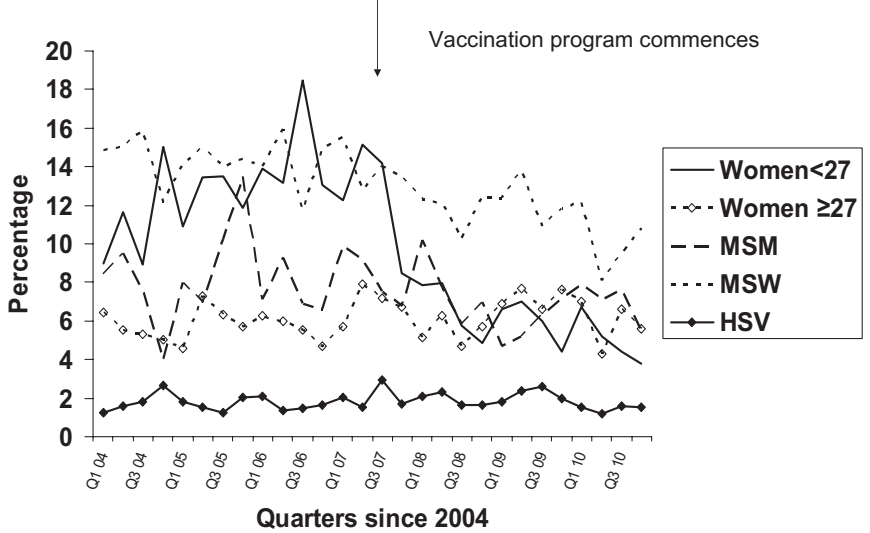

Abstract P5-S3.04 Figure 1 Presentations with Warts Quarters 2004 to end 2010.
Methods Diagnoses for all new patients were recorded and the proportion of patients diagnosed with warts was calculated for exclusively homosexual (MSM) and heterosexual men (MSW) and, for women according to their eligibility for vaccination. Women aged $\geq 27$ years in 2007 were too old for the vaccine program. Changes in these proportions are reported from 2004 to the end of 2010 (to be updated to 30 June 2011).

Results 53088 new patients were seen from 2004 to the end of 2010 and 5191 were diagnosed with warts. The proportion of patients diagnosed with warts fell from $11.2 \%$ (95\% CI 9.8 to $12.8 \%$ ) in the third quarter of 2007 to $7.3 \%$ (95\% CI 6.2 to $8.4 \%$ ) in the final quarter of 2010. Women aged $<27$ years in 2007 experienced the greatest quarterly reduction $(\mathrm{OR}$ per quarter $(\mathrm{OR}) 0.93, \mathrm{p}<0.001)$ to a proportion of $3.8 \%$ seen with warts in the 4 th quarter of 2010. There was no significant change in women $\geq 27$ years (OR 1.0, $\mathrm{p}=0.9$ ) or in homosexual men (OR 0.99, $\mathrm{p}=0.54$ ) and a small reduction in heterosexual men (OR $0.98, p=0.005)$. The proportion of younger women diagnosed with warts is continuing to fall, compared to other groups and to the proportions diagnosed with first episode genital herpes (Abstract P5-S3.04 figure 1).

Conclusions The substantial decline in genital warts continues in young Australian women, 3 years after the roll-out of the HPV vaccination program. Heterosexual men are experiencing a smaller, but significant decline.

\section{P5-S3.05 A CLINICAL PRACTICE INTERVENTION TO INCREASE CHLAMYDIAL SCREENING: SUSTAINING THE GAIN AND TRANSLATING INTO PRACTICE 4 YEARS LATER}

doi:10.1136/sextrans-2011-050108.541

K Tebb, M A Shafer. University of California, San Francisco, USA

Background Chlamydia trachomatis (CT) continues to be a major public health problem among sexually active adolescent and young adult females. Although annual CT screening is recommended, actual rates remain low. System-based interventions are an effective approach to improve clinical practice including CT screening.

Objective There are two objectives: (1) To describe the sustainability of a systems-based, clinical practice improvement intervention to increase urine-based CT screening among adolescent girls (14-18 years old) during routine check-ups in a paediatric clinic setting (2) To describe and evaluate the translation of this intervention into control clinics. 
Methods The study randomised 10 paediatric clinics (5 experimental, 5 control) within a large Northern California health maintenance organization (HMO). Clinics in the experimental group received a systems-based clinical practice improvement intervention (CPI) and controls received a traditional provider education intervention. The original study took place between 2000 and 2002 (Shaferet al JAMA, 2002). After the study ended, the intervention was translated to the remaining clinics including to the five controls. Data were tracked for four additional years (2003-2006). The proportion of 14-18-year-old girls who had sexual intercourse and who were screened for CT during their routine checkups was calculated using the same methodology as the original study. We assessed changes in the rate variable over time, within sites and between the intervention and control groups using linear mixed effects models with random intercepts.

Results The average screening rate in the intervention group was sustained at an average of $60 \%$ during the 4 -year follow-up period (CI 0.41 to 0.79 ) with no significant increases over time. Prior to translation activities, the proportion screened in controls was $21 \%$. After translation activities, the control group exhibited statistically significant linear and quadratic effects of time $\left(p=0.0019\right.$ by Wald $\chi^{2}$ test). The estimated rate for the controls was 0.42 (95\% CI 0.25 to 0.59 ) at time 1 , increased to a maximum of 0.69 (95\% CI 0.55 to $0.83)$ at year 2.5 , then declined to $0.52(95 \%$ CI 0.35 to 0.70$)$ at the end of year 4 .

Conclusions This CPI systems intervention was both translatable and sustainable to other paediatric clinics within this $\mathrm{HMO}$.

\section{Health services and policy poster session 4: innovation \\ P5-S4.01 TRACNET: SUSTAINING MHEALTH AT SCALE IN RWANDA}

doi:10.1136/sextrans-2011-050108.542

J P Nyemazi. Ministry of Health, Kigali, Rwanda

TRACnet is an mHealth system that has been supporting the national HIV/AIDS program of Rwanda since 2004 with funding and technical support from the US Centers for Disease Control. The longest continuously operating national $\mathrm{mHealth}$ system in Africa, TRACnet was built by Voxiva for and with the Government of Rwanda and the Center for Treatment and Research on AIDS, Malaria, Tuberculosis and Other Epidemic diseases (TRAC Plus). Today, more than 350 facilities offering ART, VCT and PMTCT submit monthly program indicators and receive lab results using the web or mobile phones. $91 \%$ of routine reports use mobile phones to report; the rest use the Internet. Using Rwanda's burgeoning mobile infrastructure, TRACnet has achieved 67 months of continuous nationwide routine reporting of data on ART care since 2004. In addition, more than 100000 patients on care and treatment have been registered. The system includes $>8000$ reports submitted, $>98 \%$ reporting rate, 1200 users, $>100000$ patients registered The Ministry of Health and TRACPlus are also extending the system to incorporate integrated disease surveillance and response. Voxiva has an active partnership with MTN which is providing a national tollfree line to TRACnet, GPRS SIM cards and free access for users. Data collected by TRACnet is used for a variety of purposes, for example: to monitor program expansion and progress; drive performance-based incentive payments to health center; deliver lab results to facilitate early infant diagnosis; and provide donor reporting. Sustainability is a major focus of the TRACnet program. An innovative framework that addresses all components of operating and maintaining a national enterprise system guides capacity building efforts in collaboration with Rwanda's School of Public Health. This abstracts presents the experience of 6 years of continuous operation of an mHealth solution at national scale.

\section{P5-S4.02 ROUTINE HIV TESTING OF FAMILY MEMBERS OF HOSPITALISED PATIENTS: A NOVEL APPROACH TO HIV TESTING IN NIGERIA, SUB SAHARA AFRICA}

doi:10.1136/sextrans-2011-050108.543

${ }^{1} \mathrm{O}$ Busari, ${ }^{2} \mathrm{~A}$ Adeyemi, ${ }^{3} \mathrm{M}$ Nakayima. ${ }^{1}$ Federal Medical Centre, Ido-Ekiti, Nigeria; ${ }^{2}$ Family Health International, Abuja, Nigeria; ${ }^{3}$ The AIDS Support Organization, Masaka, Uganda

Background In traditional African setting, family members are closely knitted and provide effective social and supportive care for their loved ones during admission in hospitals. HIV testing for family members of HIV positive (HIV+) patients may enhance disclosure of status of spouses, encourage family social support and improve access to HIV services. The objective was to evaluate the approach of HIV testing of family members of both HIV + and HIV - patients on admission in a large national HIV-treatment centre in Nigeria, West Africa.

Methods This was a prospective study in which HIV testing was offered to consented family members of HIV + and HIV - patients on admission between January 2009 and June 2010.The family members included spouses, children of patients, parents of paediatric patients and other family members. Analysis was done in frequencies and percentages.

Results 2829 family members of 3284 patients were tested. The details are: spouses, 339 (12\%); fathers, 255 (9\%); mothers, 1442 (51\%); and others family members, 792 (28\%). 2630 (93\%) of testers were first timers. Most of the testers ( $97 \%$ ) had post-test counselling. Overall HIV prevalence was 14\%: 7\% among spouses; $11 \%$ and $7 \%$ among mothers and fathers respectively; and $4 \%$ among other family members. Discordant status occurred in 19\% of couples tested.

Conclusion The results indicate that routine HIV testing of family members of patients on admission is a strategy for identification of large number of HIV infected persons. This method is not only innovative, but also a novel approach effective for scaling up of access to HIV prevention, care and treatment services in sub-Sahara Africa

\section{P5-S4.03 SYSTEMATIC SELECTION OF SCREENING PARTICIPANTS BY RISK SCORE IN CHLAMYDIA SCREENING PROGRAMME IS FEASIBLE AND EFFECTIVE}

doi:10.1136/sextrans-2011-050108.544

${ }^{1} \mathrm{C} J$ P A Hoebe, ${ }^{1} \mathrm{E}$ E H G Brouwers, ${ }^{2} \mathrm{~J}$ E A M van Bergen, ${ }^{3} \mathrm{~J} \mathrm{~S}$ A Fennema, ${ }^{4} \mathrm{H}$ M Gotz, ${ }^{3} \mathrm{R}$ H Koekenbier, ${ }^{5} \mathrm{E}$ L M Op de Coul, ${ }^{2} \mathrm{~L}$ Pars, ${ }^{4} \mathrm{~S}$ M van Ravensteijn, ${ }^{5}$ I V F van den Broek. 'South Limburg Public Health Service, Sittard-Geleen, Netherlands; ${ }^{2}$ STI AIDS Netherlands, Amsterdam, Netherlands; ${ }^{3}$ Amsterdam Health Service, Amsterdam, Netherlands; ${ }^{4}$ Rotterdam Rijnmond Public Health Service, Rotterdam, Netherlands, ${ }^{5}$ Centre for Infectious Disease Control, National Institute of Public Health and the Environment, Bilthoven, Netherlands

Background Systematic screening for Chlamydia trachomatis (Ct) by individual invites ensures general reach, but is less (cost) effective, as it includes people at no or low risk. Selective systematic screening can overcome this. In a large-scale Chlamydia Screening programme in the Netherlands selection by risk score was applied in one region where relatively low prevalence was expected. Here we show the effect of selection on participation, positivity and acceptability in three srceening rounds.

Methods Invitees were alerted by personal letter to login to http:// www.chlamydiatest.nl/ and fill in an 8-item questionnaire before a test could be requested. Questions, based on a prediction rule assessed in a pilot, addressed age, place of residence, education, ethnicity, symptoms, condom use and sex partners. Answers yielded different points accumulating in a risk score. Only invitees with 